\title{
Tumor-suppressive microRNA-let-7a inhibits cell proliferation via targeting of E2F2 in osteosarcoma cells
}

\author{
TATSUYA IWASAKI, KAZUHIRO TANAKA, MASANORI KAWANO, ICHIRO ITONAGA and HIROSHI TSUMURA
}

Department of Orthopaedic Surgery, Faculty of Medicine, Oita University, Oita 879-5593, Japan

Received November 21, 2014; Accepted January 12, 2015

DOI: 10.3892/ijo.2015.2867

\begin{abstract}
MicroRNAs (miRNAs) regulate cell proliferation and differentiation by silencing gene expression at the post-transcriptional level; moreover, by binding to the complementary sequences within mRNAs in cancer cells, these small non-coding RNA molecules can function as tumor suppressors or oncogenes. Recently, the dysregulation of miRNA expression has been found to be associated with increased tumorigenicity and poor prognosis in several cancer types, including osteosarcoma (OS). To identify potential oncogenic factors in OS, we analyzed changes in the expression profile of miRNAs and its downstream mRNAs in five OS cell lines and human mesenchymal stem cells (hMSCs) by a microarray-based approach. The expression of an miRNA-let-7a was significantly downregulated and E2F2 was significantly upregulated in all tested OS cells compared with hMSCs. When let-7a was transfected into OS cell lines, the expression of E2F2 in the cells was greatly suppressed, suggesting that E2F2 is a target of miRNA-let-7a in OS cells. The transfection of let-7a further inhibited cell cycle progression and proliferation of OS cells. In addition, let-7a overexpression in OS cells significantly suppressed the tumor growth in vivo. The present study demonstrates the novel mechanism that regulates E2F2 expression via miRNA-let-7a in OS cells. Because E2F2 is pivotal in promoting cell growth through the regulation of several genes, our results might facilitate the development of new therapeutic targets for the treatment of OS.
\end{abstract}

\section{Introduction}

Osteosarcoma (OS) is the most common primary sarcoma of bone and the leading cause of cancer death among adolescents and young adults (1). Most variants of OS are extremely aggressive, and are characterized by their rapid growth and

Correspondence to: Dr Kazuhiro Tanaka, Department of Orthopaedic Surgery, Faculty of Medicine, Oita University, Oita 879-5593, Japan E-mail:ktanaka@oita-u.ac.jp

Key words: let-7a, E2F2, cell cycle, apoptosis, osteosarcoma early development of distant metastasis, mostly to the lungs and bones. The 5-year survival rate of OS patients has significantly improved over the past decades to $\sim 60-70 \%$ since the introduction of combinatorial chemotherapy (2). Despite attempts to further improve the disease-free survival rates for patients responding poorly to therapy by administering more intensive therapies, it has been estimated that $30 \%$ of children diagnosed with OS do not survive beyond 5 years and $<50 \%$ live more than 10 years $(3,4)$.

MicroRNAs (miRNAs) are evolutionarily conserved, small, non-coding RNA molecules of 22 -nucleotides in length that can specifically interact with the 3'-untranslated region (3'UTR) of targeted messenger RNA (mRNA), inhibit mRNA translation, and lead to mRNA cleavage and degradation. miRNAs have the potential to regulate various critical biological processes (5). Several reports have suggested that the deregulation of miRNA is a hallmark of cancer $(6,7)$, probably because miRNAs act as either tumor suppressors or oncogenes that regulate tumor development, proliferation, invasion and metastasis (8-11). Let-7 was first described in Caenorhabditis elegans as a heterochronic switch gene (12). The let-7 expression levels were found to be lower in the lung cancer tissues than in the normal lung tissues, and let-7 can prevent the cellular proliferation via downregulation of oncogenes such as RAS, HMGA2, MYC, DICER and LIN28 (13-16). However, the role of let-7 in the proliferation of OS cells remains unclear.

The E2F family of transcription factors is the downstream effector of the retinoblastoma $(\mathrm{Rb})$ protein pathway. Eight $\mathrm{E} 2 \mathrm{~F}$ family members have been identified and divided into two groups in cell cycle control: E2F1-3 function mainly as activators, while E2F4 and E2F5 primarily act as repressors $(17,18)$. In addition, E2Fs modulate diverse cellular functions such as DNA repair, differentiation and development $(19,20)$. E2F2 plays a central role in the regulation of G1/S transition and cell cycle progression through the $\mathrm{S}$ phase, subsequently promoting the cellular transformation (21). Although several miRNAs have been found to target E2F2, including let-7a, miR-17-92 cluster (22) and miR-24 (23), the correlation of E2F2 expression and miRNA in OS cells is completely unknown.

In the present study, we used a genome-wide expression array to analyze both miRNAs and mRNAs in five human OS cell lines and human mesenchymal stem cells (hMSCs). The expression of let-7a was decreased, whereas that of E2F2 was increased in all five OS cell lines compared with the hMSCs. Based on the inverse correlation between let-7a and E2F2 
expression, we hypothesize that the effect of E2F2 in OS cells may be mediated, at least in part, through let-7a expression. We aimed to assess whether the expression of E2F2 is regulated by let-7a, and whether the pathway plays a role in the tumorigenesis of OS cells.

\section{Materials and methods}

Cell lines. The human OS cell lines, HOS, SaOS and MG-63, were obtained from RIKEN Cell Bank (Tsukuba, Japan), and NY and Hu09 were obtained from the Japanese Collection of Research Bioresources Cell Bank (JCRB, Osaka, Japan). hMSCs were purchased from Takara Biotechnology (Otsu, Japan). The genotype and phenotype of each cell line was authenticated by the respective source company. HOS cells were grown in minimal essential medium (MEM) supplemented with $10 \%$ fetal bovine serum (FBS; Invitrogen, Grand Island, NY, USA) and $0.1 \mathrm{mmol} / 1$ non-essential amino acids (NEAA). SaOS, MG-63 and NY cells were cultured in a high-glucose medium, Dulbecco's modified Eagle's medium (DMEM; Invitrogen) supplemented with 10\% FBS and 1\% penicillin and streptomycin. The $\mathrm{Hu}$ cells were cultured in Roswell Park Memorial Institute medium (RPMI)-1640 (Invitrogen) supplemented with 10\% FBS. hMSCs were cultured with the Chemically Defined Mesenchymal Stem Cell Basal Medium (MSCBM-CD) with MSCGM-CD SingleQuots (Takara Biotechnology). The cells were maintained at $37^{\circ} \mathrm{C}$ under $5 \% \mathrm{CO}_{2}$, and passaged every 2-3 days.

RNA isolation. mRNAs were prepared from the triplicated cell cultures by using the RNeasy kit (Qiagen, Valencia, CA, USA) according to the manufacturer's instruction. The RNA quality was assessed, before labeling, by using the RNA 6000 Nano kit and Bioanalyzer 2100 (Agilent Technologies Inc., Santa Clara, CA, USA).

Genome-wide miRNA expression microarray. The GeneChip miRNA 3.0 array (Affymetrix, Santa Clara, CA, USA) was used for miRNA expression profiling of five OS cell lines and hMSCs. Small RNA (1 $\mu \mathrm{g})$ including miRNA from each sample was labeled with biotin using the FlashTag Biotin HSR kit (Genisphere LLC, Hatfield, PA, USA). Array hybridization, washing, and scanning of the slides were performed in accordance with the manufacturer's instructions. The data were extracted from the images, quantile-normalized, summarized (median polish), and $\log _{2}$-transformed with miRNA QC software (Affymetrix). The GeneSpring GX 11.0 (Agilent Technologies) was used to analyze the array results. Analysis of variance (ANOVA) was used to determine the significant difference in the probe sets between the two groups. The gene list was filtered with a fold-change cut-off of 2, resulting in the output of list with genes that have significant differential expression at $\geq 2$-fold differences. Pathway analysis was performed using the KEGG pathway database (http://www. genome.jp/kegg/pathway.html).

Analysis of mRNA expression by cDNA arrays. The GeneChip Genome Human Genome U133 Plus 2.0 array (Affymetrix) was used for mRNA expression profiling of five OS cell lines and hMSCs. Biotinylated cRNA was synthesized from total
RNA by using the 3' IVT Express kit (Affymetrix) according to the manufacturer's instructions. Briefly, double-stranded cDNA was generated by reverse transcription from $1 \mathrm{ng}$ of total RNA using an oligo(dT) primer bearing a T7 promoter. The double-strand cDNA was used as a template for in vitro transcription to generate biotin-labeled cRNA. After fragmentation, $12.5 \mu \mathrm{g}$ of cRNA were hybridized to the GeneChip array for $16 \mathrm{~h}$. The arrays were washed and stained using the GeneChip Fluidics Station 450 (Affymetrix) and then scanned with the GeneChip Scanner 3000 (Affymetrix). The entire experiment was performed twice. Array hybridization, washing, and scanning of the slides were performed according to the manufacturer's instructions. The microarray numerical values were analyzed using the GeneSpring GX 11.0 software according to the RAM16 Algorithm (12925520): quantile normalization, filter by flags (detected), and filter by expression on the normalized data (20.0-100.0th percentile). ANOVA was used to determine the significant difference between the two groups. The gene list was filtered to include genes showing at least a 2 -fold change in expression.

Target prediction of miRNAs. Basic Local Alignment Search Tool (BLAST) and TargetScan 6.0 (microRNA.org) were used to search for the predicted target genes of miRNAs.

Mature miRNA transfection. One day prior to transfection, the cells were seeded onto 6 -well plates $\left(5 \times 10^{4}\right.$ cells/well $)$ and incubated with the complete medium without antibiotics ( $2 \mathrm{ml} /$ well). The transfection of let-7a-2-3p mimic or negative control (NC) miRNA (Invitrogen) was performed using Lipofectamine 2000 (Invitrogen) in antibiotic-free OptiMEM (Invitrogen) according to the manufacturer's instructions. After 48-h of incubation following transfection, the cells were harvested and processed for further analysis. The experiment were repeated three times.

Knockdown of E2F2 expression using siRNA. siRNA oligonucleotides targeting E2F2 mRNA was purchased from Ambion (Tokyo, Japan) and the Mission siRNA Universal Negative Control was purchased from Sigma-Aldrich (Osaka, Japan). siRNAs were transfected into MG63 and SaOS cells using Lipofectamine 2000 according to the manufacturer's instructions. The cells were harvested $48 \mathrm{~h}$ after transfection and then subjected to various analyses. The experiment was repeated three times.

Cell proliferation assay. The cells were plated into 6-well plates $\left(5 \times 10^{4}\right.$ cells/well) and transfected with or without let7a-2-3p mimic, NC miRNA or E2F2 siRNA. Next, the cells were incubated in antibiotic-free Opti-MEM. After 48-h cultivation, the cells were counted by using the TC10 Automated Cell Counter (Bio-Rad Laboratories).

Western blot analysis. Total cellular protein $(15 \mu \mathrm{g})$ was resolved on a precast $10 \%$ Tris- $\mathrm{HCl}$ Criterion 10 -well gel (Bio-Rad Laboratories) at $200 \mathrm{~V}(300 \mathrm{~mA}$ ) for $30 \mathrm{~min}$. The gel was wet-transferred onto a polyvinylidene difluoride (PVDF) membrane for $1 \mathrm{~h}$ and blocked with phosphate-buffered saline (PBS) solution containing Tween-20 (PBST) and 5\% instant dry non-fat milk for $30 \mathrm{~min}$ at room temperature. Polyclonal 
rabbit anti-human antibodies against E2F2 (ab65222) and $\beta$-actin (ab16039) proteins were obtained from Abcam (Cambridge, UK). The immunocomplexes were visualized using horseradish peroxidase-conjugated anti-rabbit immunoglobulin $\mathrm{G}$ antibodies (GE Healthcare, Tokyo, Japan) and the blots were developed by using the ECL Plus system (GE Healthcare) attached with a ChemiDoc camera (ImageQuant LAS 4000 mini; GE Healthcare). The quantification of western blot signals was performed by the densitometry using ImageQuant TL software (GE Healthcare). All experiments were repeated at least three times.

Cell cycle analysis. For cell cycle analysis, the cells were stained with propidium iodide using the Cycletest Plus DNA reagent kit (BD Biosciences) according to the manufacturer's instructions, and the cell cycle distribution was analyzed by the FACSVerse flow cytometer (BD Biosciences). The percentages of cells in the G0/G1, S and G2/M phases were counted and compared. All experiments were performed in triplicates.

Apoptosis assay. The changes in the expression of apoptotic proteins were determined by western blot analysis using polyclonal rabbit anti-human antibodies against PAR/poly (ADP-ribose) polymerase (PARP) (\#9542) and cleaved PARP (\#9541) (Cell Signaling Technology, Tokyo, Japan) as an index of apoptosis. The quantification of cell death was determined by fluorescence-activated cell sorting (FACS) using the Annexin V-FITC Apoptosis Detection kit (BD Biosciences) according to the manufacturer's instructions. Briefly, $1 \times 10^{6}$ MG63 cells were seeded and incubated for $24 \mathrm{~h}$, then let-7a-2-3p mimic or siRNA for E2F2 was added to the cells and incubated for $48 \mathrm{~h}$. The cells were washed with PBS, suspended in Annexin V binding buffer, added to an Annexin V-FITC/PI solution, and incubated for $20 \mathrm{~min}$ at room temperature. The samples were analyzed by the FACSVerse flow cytometer using FACSuite Analysis software (BD Biosciences). The MG63 cells treated with doxorubicin at $40 \mathrm{mg} / \mathrm{ml}$ for $20 \mathrm{~h}$ were used as a positive control for apoptosis.

In vivo tumor-bearing nude mouse model. The experimental metastasis model was established by injecting $1 \times 10^{6}$ cells transfected with let-7a miRNA suspended in $100 \mathrm{ml}$ of normal saline in the gluteal region of nude mice. The mice were divided into three groups: i) untreated control $(n=7)$; ii) transfected with NC-miRNA ( $=7)$; and ii) transfected with let-7a miRNA mimic $(n=7)$. All mice were fed with the standard diet and their weight was monitored; the mice were sacrificed 6 weeks after cell inoculation. Tumor size in mice was measured in two perpendicular dimensions parallel with the surface and the depth of the tumor using a caliper. The tumor volume of the lung nodule was estimated using the formula: $\pi \mathrm{x}$ long axis $\mathrm{x}$ short axis $\mathrm{x}$ short axis) $/ 6$. All experiments were performed under the guidelines for animal experiments as stipulated by the Oita University Graduate School of Medical Science.

Statistical analysis. Statistical analysis was performed using SPSS 18.0 (SPSS Japan Inc., Tokyo, Japan). Two-tailed Student's t-test was used for the analysis of continuous variables. We determined the differences among the three groups by using a non-repeated measures ANOVA and Scheffe's test.
The results were expressed as the mean \pm standard deviation, and $\mathrm{P}<0.01$ was considered statistically significant.

\section{Results}

Downregulation of let-7a expression in the OS cell lines. The genome-wide miRNA expression profiling of five OS cell lines was performed to identify miRNAs specifically expressed in the OS cells. The array analysis revealed that 435 miRNAs in the OS cells showed a $>2.0$-fold change in expression compared with hMSCs (Fig. 1A). Among 435 miRNAs, 186 were significantly upregulated, whereas 170 were significantly downregulated in all tested OS cells compared with hMSCs. The remaining 79 miRNAs were upregulated or downregulated among the five OS cell lines. The expression of let-7a decreased by $11.25-21.68$-fold in the OS cell lines compared with hMSCs.

Upregulation of E2F2 expression in OS cell lines. The cDNA array analysis demonstrated that the expressions of 761 mRNAs were significantly changed between the five OS cell lines and hMSCs (Fig. 1B). We found that 123 genes were significantly upregulated, 562 genes were significantly downregulated, and the remaining 194 genes were upregulated or downregulated in the five OS cell lines compared with hMSCs. Furthermore, the expression of E2F2 was increased by 2.36-4.27-fold in all five OS cell lines compared with the hMSCs.

Let-7a has several predictive binding sites in the 3'UTR of $E 2 F 2$. A considerable complementarity between sequences within the seed regions of let-7a-2-star and sequences in the 3'UTR of E2F2 was identified, using the algorithms in BLAST and TargetScan. The results suggested that let-7a might affect the expression of E2F2 genes by binding to 3'UTRs of E2F2. The analysis predicted that the targeting region of E2F2 3'UTR binding hsa-let-7a-2-star is at position 972-994 (Fig. 2A), 10761098 (Fig. 2B), 1487-1509 (Fig. 2C), 1988-2000 (Fig. 2D) and 3066-3088 (Fig. 2E).

Inhibition of E2F2 expression by let-7a miRNA and E2F2 siRNA. To examine the correlation between let-7a and E2F2 in the OS cells, let-7a miRNA was transfected into the MG63 cells. Western blot analysis revealed that the expression levels of E2F2 dramatically decreased in the let-7a-transfected cells compared with untreated or NC oligo-transfected cells (Fig. 3A). The protein expression levels of E2F2 in the let-7a-transfected $(40 \mathrm{nM})$ cells were reduced to $40 \%$ of that in the control cells $(\mathrm{P}<0.01)$ (Fig. 3B). To further confirm the effects of E2F2 on the growth of OS cells, transfection with siRNA targeting E2F2 was performed. Although the expression level of E2F2 protein in the cells transfected with $\mathrm{NC}$ siRNA was not significantly affected, the level in the cells transfected with E2F2 siRNA was significantly reduced, as determined by western blotting (Fig. 3C). Compared to the control cells (100\%), E2F2 siRNA (40 nM)-transfected cells exhibited 34\% lower E2F2 expression level ( $\mathrm{P}<0.01)$ (Fig. 3D).

Suppression of OS cell growth by transfection of let-7a miRNA and E2F2 siRNA. E2F2 is known to play important roles in the regulation of cell proliferation. Since the transfection of let-7a 

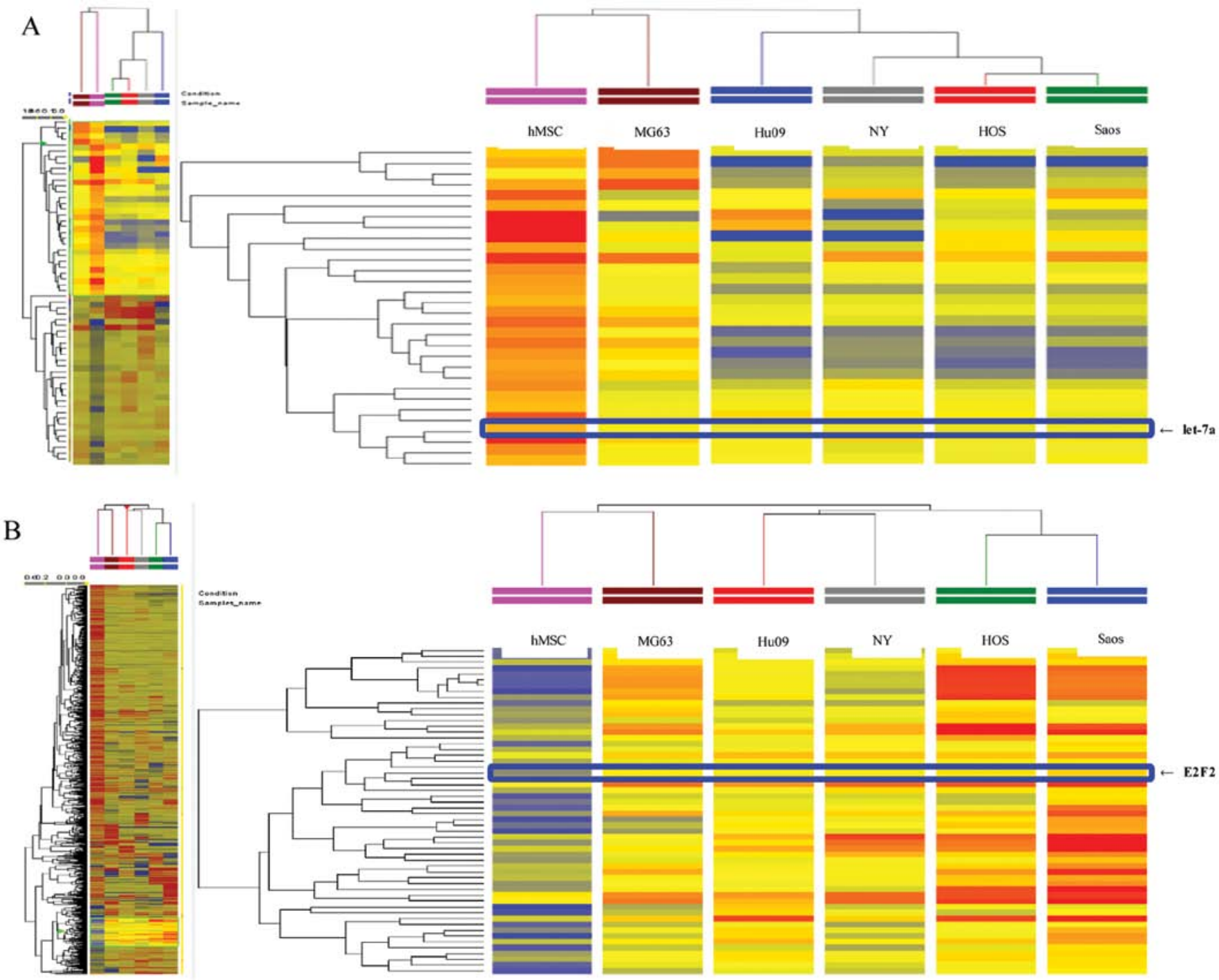

Figure 1. (A) Heat map of genome-wide miRNA profile in five OS cell lines and hMSCs. Let-7a is downregulated in all five OS cell lines. (B) Whole-genome mRNA profile and extraction of E2F2 and the related genes followed the same pathways in OS cells and hMSCs.

resulted in the reduction of $\mathrm{E} 2 \mathrm{~F} 2$ expression, we next examined the effects of let-7a on the proliferation of OS cells. The cell growth of MG63 (Fig. 4A) and Saos (Fig. 4B) was inhibited by transfection of let-7a, as determined by cell counting in comparison with untreated and NC-miRNA-transfected cells $48 \mathrm{~h}$ after transfection. Like let-7a miRNA-transfected cells, E2F2 siRNA-transfected MG63 (Fig. 4C) and Saos (Fig. 4D) cells showed significant inhibition of cell proliferation compared with untreated and NC siRNA-transfected cells.

Induction of cell cycle arrest at the G0/G1 phase by let-7a. Since the introduction of let-7a significantly inhibited cell proliferation of OS cell lines, we hypothesized that let-7a might induce the cell cycle arrest and/or apoptosis of the cells. To monitor the cell cycle distributions, FACS analyses were performed by using let-7a miRNA- and E2F2 siRNA-transfected cells (Fig. 5A-D). Both in let-7a- and E2F2 siRNA-transfected cell lines, the number of the cells in the $\mathrm{G} 2 / \mathrm{M}$ and $\mathrm{G} 0 / \mathrm{G} 1$ phase was significantly lower and higher than that in the untreated or control oligo-transfected cells, respectively (Fig. 5E). The data suggested that the restoration of let-7a and the knockdown of $\mathrm{E} 2 \mathrm{~F} 2$ leads to $\mathrm{G} 0 / \mathrm{G} 1$ arrest in the OS cells.

Subsequently, the cellular expression of PARP and its cleaved product was assayed by immunoblotting in MG63 cells and their transfectants (Fig. 5F). The cleavage of PARP protein, a marker of caspase-mediated apoptosis, was not observed in either let-7a miRNA or E2F2-siRNA transfectants or in untreated and $\mathrm{NC}$ transfectants, in marked contrast to that observed in ADM-treated (positive control) cells (Fig. 5G).

Furthermore, the flow cytometric analysis with Annexin V-FITC/PI double staining revealed no significant differences in the distribution patterns between untreated (Fig. 5H), NC miRNA- (Fig. 5I), let-7a (Fig. 5J) and E2F2 siRNA-transfected cells (Fig. 5K) compared to the positive control cells exhibiting apoptosis (Fig. 5L). The programmed cell death was not induced by let-7a miRNA or E2F2-siRNA in the MG63 OS cells.

Inhibition of tumor growth in the nude mouse xenograft model by let-7a. We next investigated the efficacy of let-7a against tumor growth in vivo. The introduction of let-7a miRNA into 


\section{Human E2F2 (NM_004091); 5228 bp}

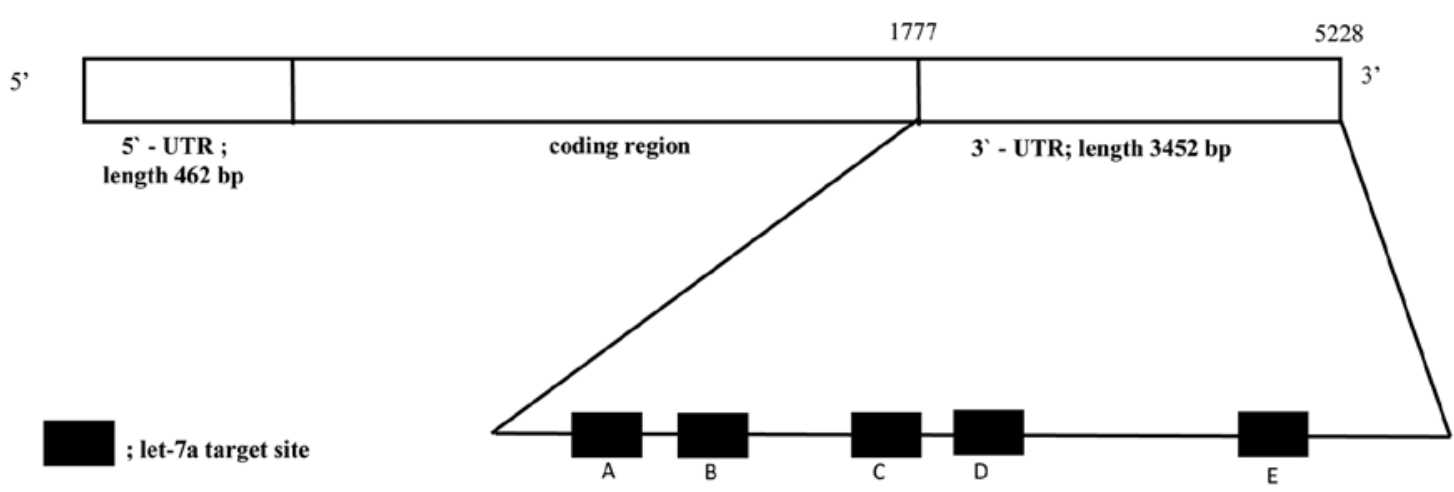

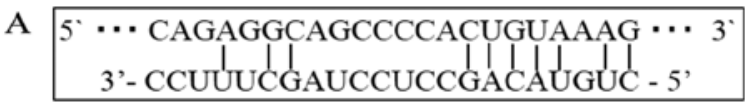

Position 972 - 994 of 3'-UTR

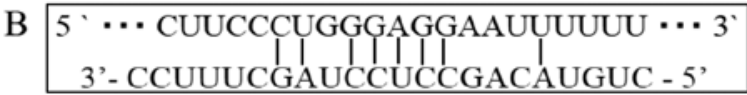

Position 1076-1098 of $3^{\prime}$-UTR

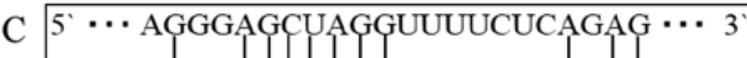

$\mathrm{D}$

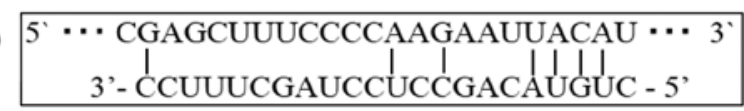

Position 1988 - 2000 of $3^{\prime}$-UTR

E

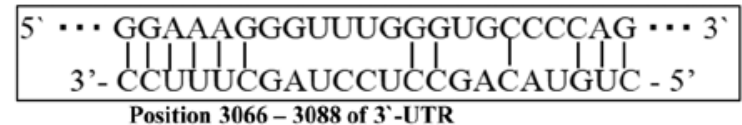

Position 1487 - 1509 of $3^{\prime}$-UTR

Figure 2. Predicted binding sites (MRE1-5) of let-7a in 3'UTR of E2F2, as aligned by TargetScan and BLAST.

A

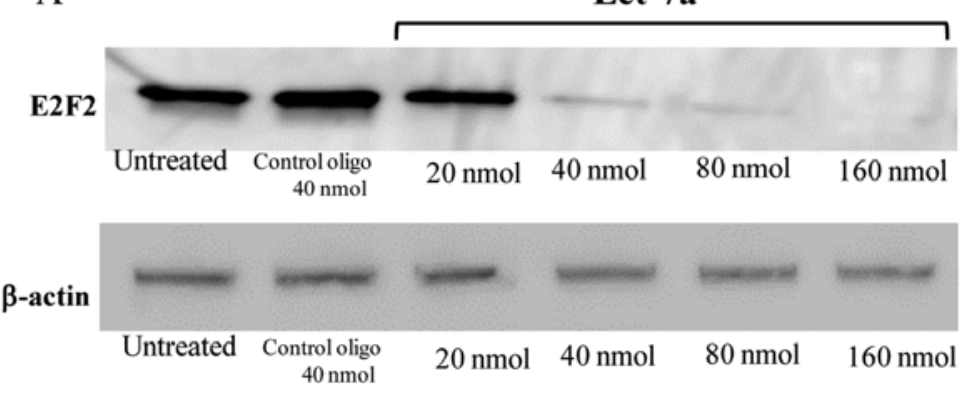

C

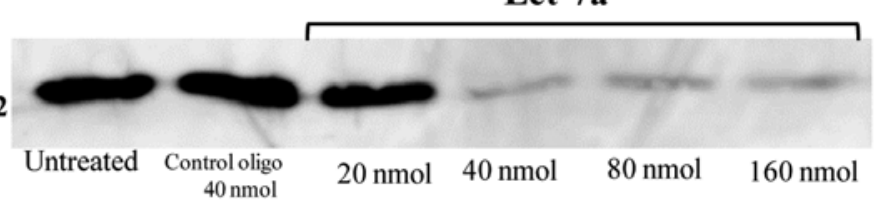

$\beta$-actin

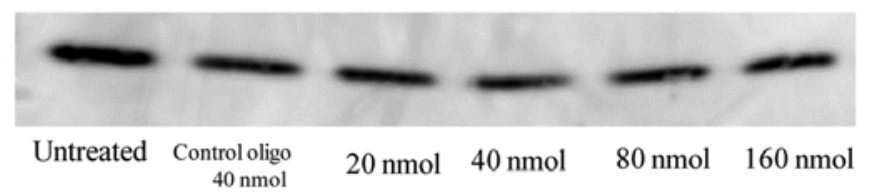

B

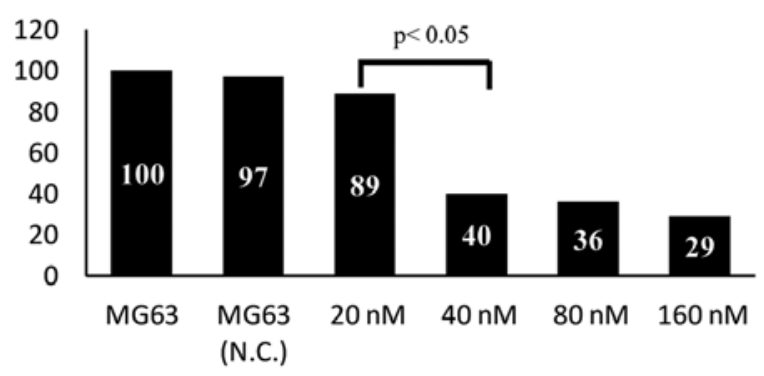

$\mathrm{D}$

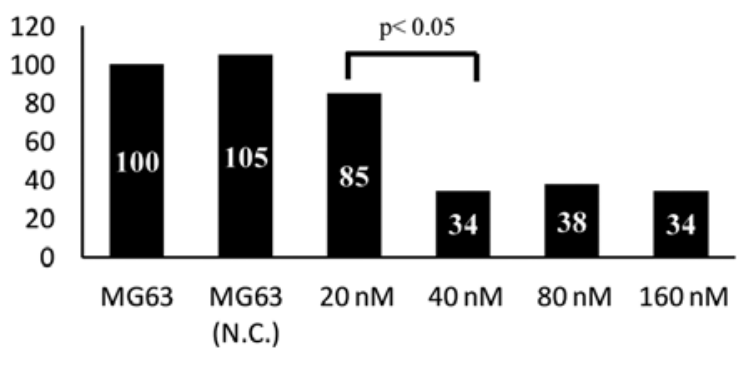

Figure 3. Cell proliferation assay performed to evaluate the antiproliferation effect of let-7a (A and B) and E2F2-siRNA (C and D) in OS cells. Error bars represent mean $\pm \mathrm{SD}$ from three independent experiments. Two-tailed Student's t-test was employed to statistically analyze the results; ${ }^{* *} \mathrm{P}<0.01$.

MG-63 cells resulted in decreased growth of subcutaneous xenografted tumors in nude mice (Fig. 6A-C). MG-63 cells transfected with let-7a showed statistically smaller tumors in mice than untreated and NC miRNA-transfected cells 

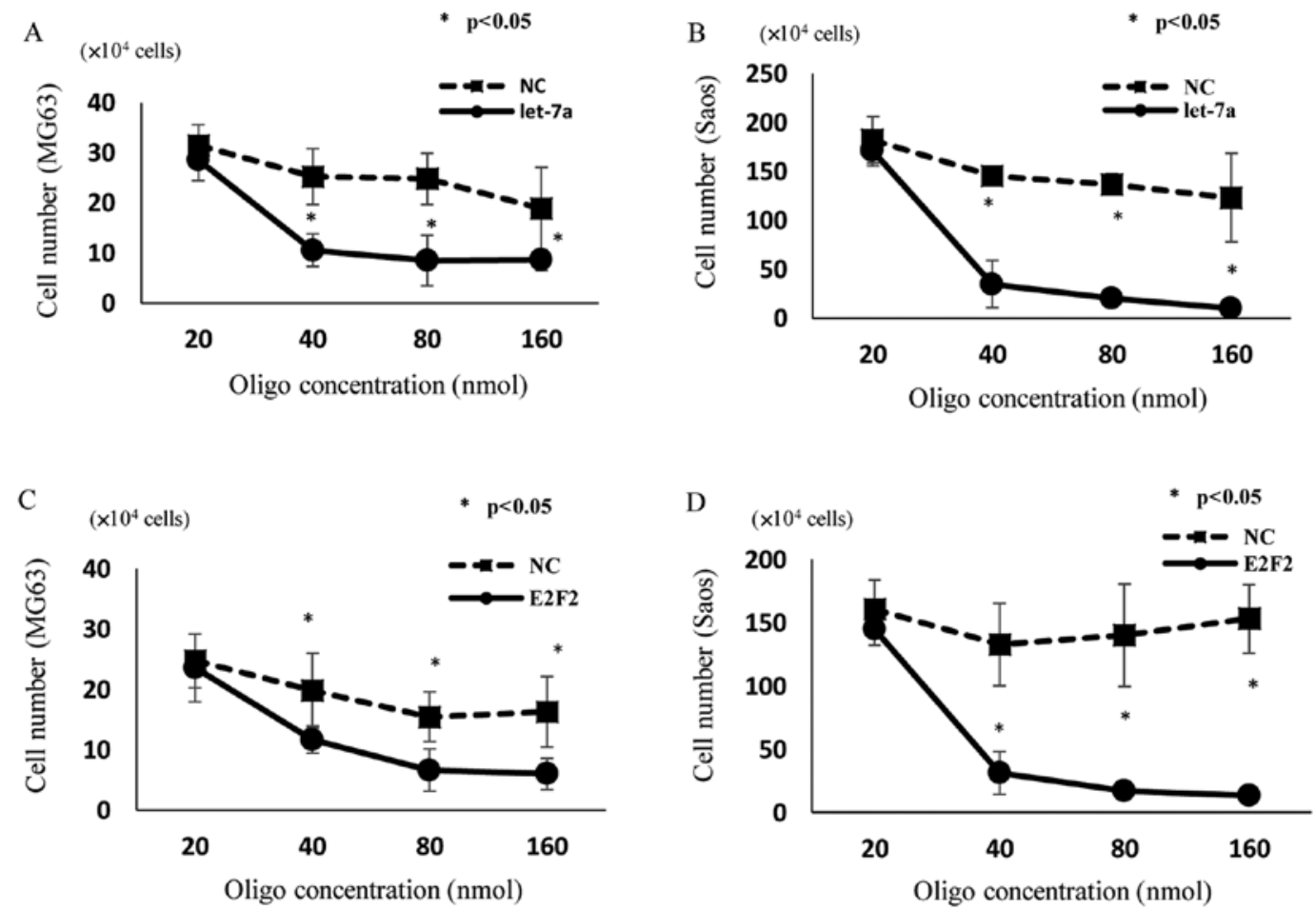

Figure 4. Silencing of E2F2 with let-7a microRNA and E2F2-siRNA in OS cells. (A) E2F2 protein is decreased in OS cells transfected with let-7a. (B) Densitometry quantification of E2F2 protein after transfection with let-7a. (C) Transfection of E2F2-siRNA in OS cells reduces the expression of E2F2 protein. (D) The quantification of $\mathrm{E} 2 \mathrm{~F} 2$ protein after transfection of $\mathrm{E} 2 \mathrm{~F} 2$-siRNA. ANOVA was performed to statistically analyze the data; ${ }^{* *} \mathrm{P}<0.01$.
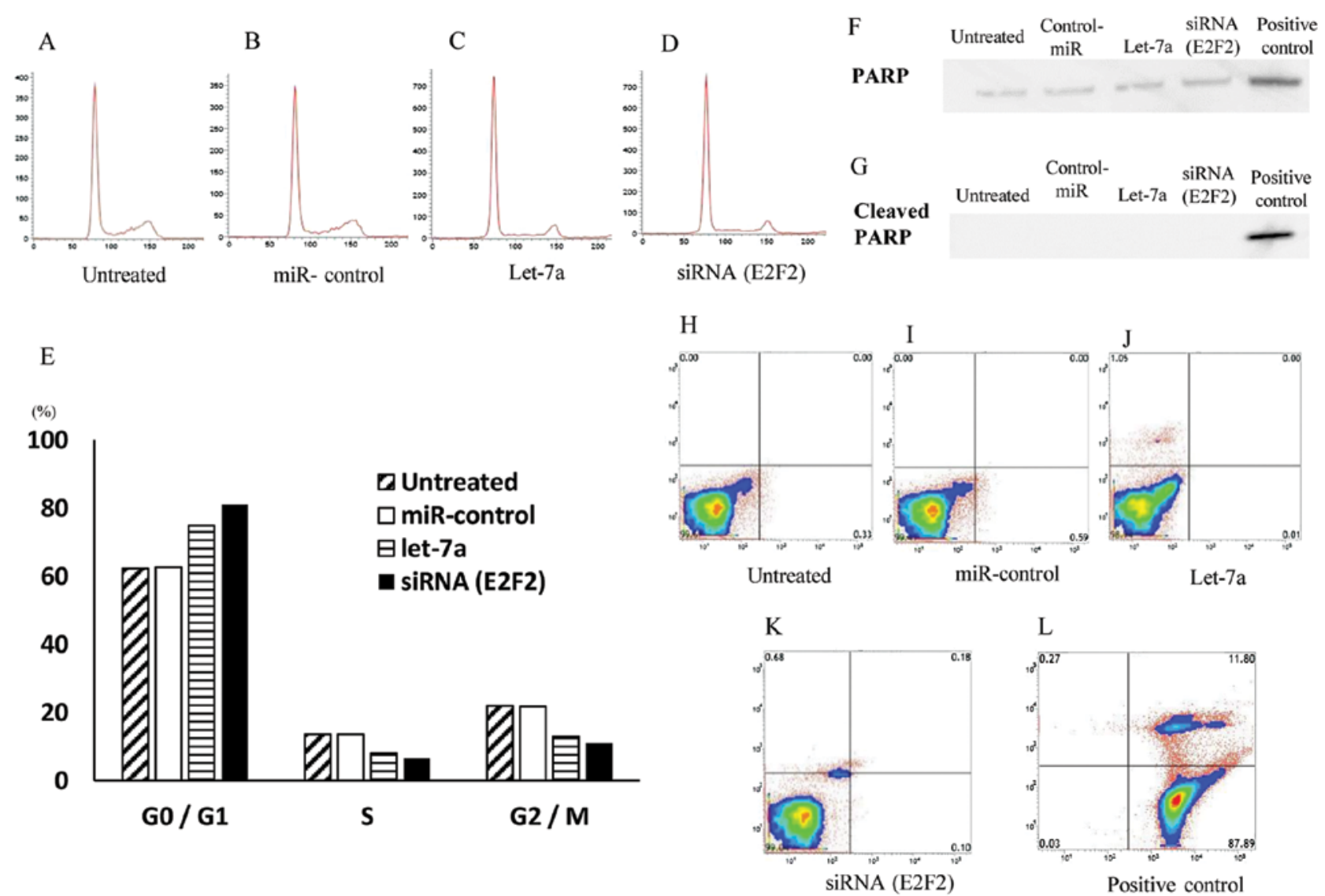

Figure 5. Effect of let-7a miRNA and E2F2-siRNA on the cell cycle in MG63 cells. The cells were treated and analyzed by flow cytometry after PI staining (A-D). Histogram shows the quantitative percentage of diploid cells (DNA content) in each cell cycle phase (E). Effects of let-7a and E2F2-siRNA on induction of apoptosis in the MG63 cells. Western blotting shows the expression of PARP (F) and its cleaved form (G). The cells were labeled with FITC Annexin V and PI (h-l). Each quadrant represents viable cells (lower left quadrant), early apoptotic cells (lower right), late or secondary necrotic cells (upper right), and primary necrotic cells (upper left). 

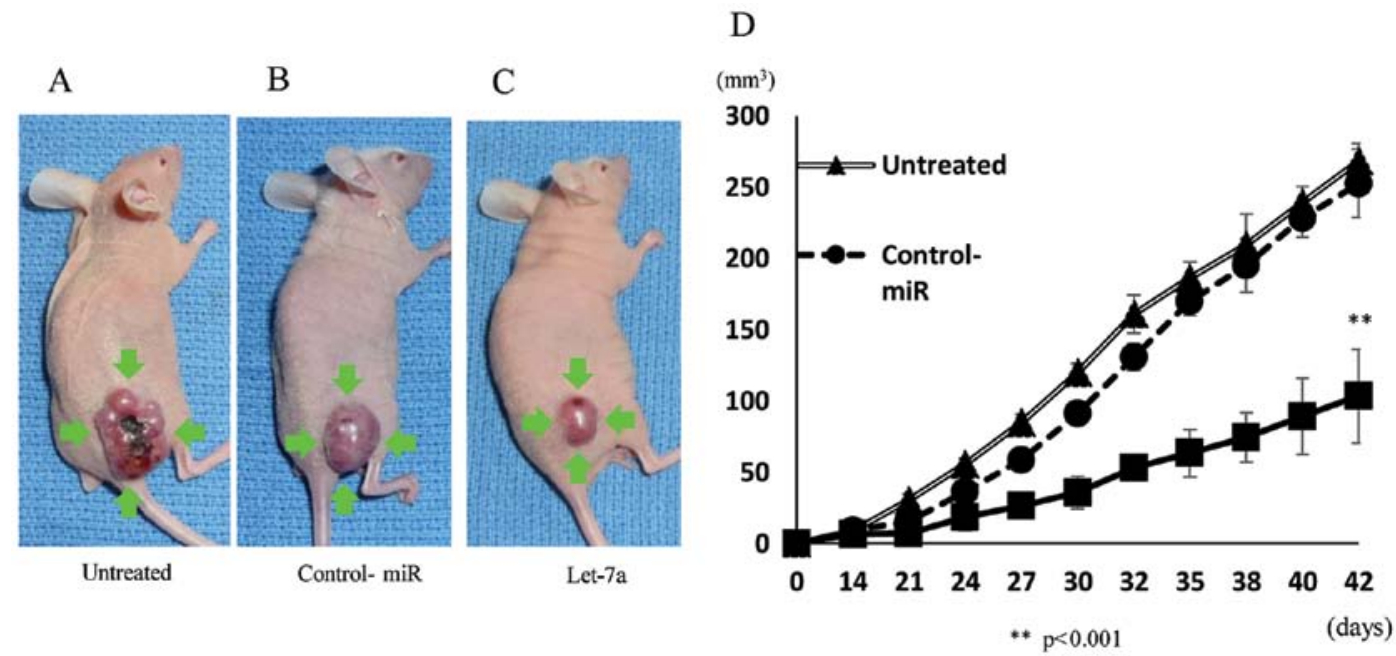

Figure 6. Let-7a suppresses the in vivo tumor growth. The three groups included (A) untreated (n=7), (B) transfection of NC-miRs (n=7), and (C) transfection of let-7a $(\mathrm{n}=7)$. Tumor volumes were measured at the indicated time-points after tumor cell inoculation $(\mathrm{D}){ }^{* * *} \mathrm{P}<0.01$.

(Fig. 6D), indicating that let-7a also inhibits the growth of OS cells in vivo.

\section{Discussion}

It has been well established that the aberrant expression of miRNAs contributes to the proliferation, invasion or metastatic behavior of human cancer (24). One miRNA is capable of targeting multiple genes and thereby globally regulating several biological processes. Moreover, the aberrant expression of miRNAs in human cancer cells causes destruction of miRNA-mediated mRNA networks. Abnormalities in these streams could influence the expression of tumorigenic proteins. To identify important miRNA-mRNA relationship in the OS cells, we performed genome-wide miRNA expression array as well as cDNA array in the same OS cells.

In the present study, the miRNA array results demonstrated that the expression of let-7a was downregulated in all five OS cell lines. Several studies have shown that the downregulation of let-7a is closely related to the abnormal potential in malignant tumors $(13,25,26)$. However, the biological roles of let-7a in OS cells have not yet been clarified. Our results indicated that the expression of let-7a was coordinately upregulated in the OS cell lines, which led to us to performing genome-wide mRNA profiling by cDNA array to detect the possible targets of let-7a in the OS cells.

The data from cDNA array analyses showed that the E2F2 mRNA expression was increased in the five OS cell lines compared with hMSCs. Furthermore, the sequence analysis suggested possible association of let-7a with 3'UTR of E2F2. E2F2 is a member of E2F family of transcription factors and has been well studied as an important regulator of the cell cycle (19). E2F2 has a strong ability to promote cell cycle progression (27), and aberrant expression of E2F2 can lead to abnormal cellular proliferation. Upregulation of E2F2 has been reported in prostate (28), breast cancer (29) and astrocytoma (30). Our data on OS cells are consistent with those from previous studies reporting that the upregulation of E2F2 may contribute to cell malignancy.
Although let-7a probably influences the expression of several genes, we focused on E2F2 as the target of let-7a in OS cells. Several target genes of let-7a have been reported, such as RAS, HMGA2, MYC, DICER and LIN28 (13-16). Our cDNA array analysis demonstrated that E2F2 was the only let-7a target gene whose expression was uniformly upregulated in all five OS cell lines, whereas the expression of other candidate genes differed among the OS cell lines. The analysis using several algorithms such as BLAST and TargetScan further suggested that E2F2 was the putative target of let-7a. Thus, we analyzed the possibility that let-7a may contribute to anticancer activities by targeting E2F2 in the OS cells.

We next examined the functions of let-7a in the regulation of its possible target gene, E2F2, and the changes in the biological characteristics in the OS cell lines. The forced elevation of let-7a levels resulted in the reduction of the expression of E2F2 protein, indicating that let-7a might function as a tumor suppressor gene in the OS cells. Dong et al (31) reported that E2F2, a cell cycle progression and cell proliferation regulator, is the direct target of let-7a in prostate cancer. Our results suggested that the same regulatory mechanism of E2F2 expression via let-7a exists in the OS cells.

Our data regarding the cell cycle showed that let-7a inhibited the proliferation of OS cells via induction of the cell cycle arrest at the G1/G0 phase. These observations are consistent with those in previous studies that demonstrated the necessity of E2F2 to bypass the normal G1/S checkpoint (32). It has been reported that $\mathrm{E} 2 \mathrm{~F} 2$ plays a central role in the regulation of G1/S transition and cellular transformation and that the inhibition of E2F2 expression results in cell cycle arrest in the G1 phase (21). Thus, we can assume that the upregulation of let-7a might affect the cell cycle progression of OS cells via let-7a-mediated control of the E2F2 expression. Notably, the downregulation of E2F2 by challenge of let-7a miRNA or siRNA against E2F2 did not induce apoptosis of OS cells, indicating that the repression of OS cell growth was acquired by cell cycle retardants.

Furthermore, the overexpression of let-7a in the OS cells resulted in the inhibition of OS tumor growth in vivo. In concor- 
dance with the data of in vitro experiments, the xenograft model of OS suggested that let-7a induction could inhibit OS cell development in vivo by targeting E2F2 expression.

In summary, the present study suggested, for the first time, the correlation of let-7a and E2F2 in OS cells. Our results provided evidence that the expression levels of let-7a in OS cells were significantly reduced and inversely correlated with the E2F2 expression levels and that let-7a plays an important role in OS cell proliferation and tumorigenesis by targeting E2F2 both in vitro and in vivo. Deregulated E2F2 activity is found in several human cancers in which E2F2 overexpression potently promotes cell growth and proliferation $(31,33)$. Our data suggest that E2F2 is one of the crucial factors that enhances tumor proliferation in OS, as other malignant tumors. Although the data presented in the present study needs to be confirmed by using clinical OS samples, information regarding the association between let-7a and E2F2 in OS cells would be beneficial for determining the underlying mechanisms of OS and may facilitate the development of novel therapeutic strategies for clinical application.

\section{Acknowledgements}

We thank Katsuhiro Hanada and Takashi Kobayashi for their helpful discussions during the present study. This study was supported in part by National Cancer Center Research and Development Fund (26-A-4), the Health and Labour Sciences Research Expenses (H26-084) from the Ministry of Health, Labour and Welfare, and the Grants-in-Aid for Scientific Research (no. 24592250) from Japan Society for the Promotion of Science.

\section{References}

1. Ottaviani G and Jaffe N: The epidemiology of osteosarcoma. Cancer Treat Res 152: 3-13, 2009.

2. Bielack SS, Kempf-Bielack B, Delling G, Exner GU, et al: Prognostic factors in high-grade osteosarcoma of the extremities or trunk: an analysis of 1,702 patients treated on neoadjuvant cooperative osteosarcoma study group protocols. J Clin Oncol 20: 776-790, 2002.

3. Kansara M and Thomas DM: Molecular pathogenesis of osteosarcoma. DNA Cell Biol 26: 1-18, 2007.

4. Marulanda GA, Henderson ER, Johnson DA, Letson GD and Cheong D: Orthopedic surgery options for the treatment of primary osteosarcoma. Cancer Control 15: 13-20, 2008.

5. Bartel DP: MicroRNAs: genomics, biogenesis, mechanism, and function. Cell 116: 281-297, 2004.

6. Calin GA, Dumitru CD, Shimizu M, et al: Frequent deletions and down-regulation of micro- RNA genes miR15 and miR16 at $13 q 14$ in chronic lymphocytic leukemia. Proc Natl Acad Sci USA 99: 15524-15529, 2002.

7. Ruvkun G: Clarifications on miRNA and cancer. Science 311: 36-37, 2006

8. Calin GA and Croce CM: MicroRNA signatures in human cancers. Nat Rev Cancer 6: 857-866, 2006.

9. Garofalo M, Di Leva G, Romano G, et al: miR-221\&222 regulate TRAIL resistance and enhance tumorigenicity through PTEN and TIMP3 downregulation. Cancer Cell 16: 498-509, 2009.

10. Acunzo M, Visone R, Romano G, et al: miR-130a targets MET and induces TRAIL-sensitivity in NSCLC by downregulating miR-221 and 222. Oncogene 31: 634-642, 2012.
11. Garofalo M, Romano G, Di Leva G, et al: EGFR and MET receptor tyrosine kinase-altered microRNA expression induces tumorigenesis and gefitinib resistance in lung cancers. Nat Med 18: 74-82, 2011.

12. Reinhart BJ, Slack FJ, Basson M, Pasquinelli AE, Bettinger JC, Rougvie AE, Horvitz HR and Ruvkun G: The 21-nucleotide let-7 RNA regulates developmental timing in Caenorhabditis elegans. Nature 403: 901-906, 2000

13. Takamizawa J, Konishi H, Yanagisawa K, et al: Reduced expression of the let-7 microRNAs in human lung cancers in association with shortened postoperative survival. Cancer Res 64: 3753-3756, 2004.

14. Johnson SM, Grosshans H, Shingara J, et al: RAS is regulated by the let-7 microRNA family. Cell 120: 635-647, 2005.

15. Lee YS and Dutta A: The tumor suppressor microRNA let-7 represses the HMGA2 oncogene. Genes Dev 21: 1025-1030, 2007.

16. Akao Y, Nakagawa $\mathrm{Y}$ and Naoe T: let-7 microRNA functions as a potential growth suppressor in human colon cancer cells. Biol Pharm Bull 29: 903-906, 2006.

17. Attwooll C, Lazzerini Denchi E and Helin K: The E2F family: specific functions and overlapping interests. EMBO J 23: 4709-4716, 2004.

18. Trimarchi JM and Lees JA: Sibling rivalry in the E2F family. Nat Rev Mol Cell Biol 3: 11-20, 2002.

19. Dimova DK and Dyson NJ: The E2F transcriptional network: old acquaintances with new faces. Oncogene 24: 2810-2826, 2005.

20. Wong JV, Dong P, Nevins JR, Mathey-Prevot B and You L: Network calisthenics: control of E2F dynamics in cell cycle entry. Cell Cycle 10: 3086-3094, 2011.

21. Helin K: Regulation of cell proliferation by the E2F transcription factors. Curr Opin Genet Dev 8: 28-35, 1998.

22. Aguda BD, Kim Y, Piper-Hunter MG, Friedman A and Marsh CB: MicroRNA regulation of a cancer network: consequences of the feedback loops involving miR-17-92, E2F, and Myc. Proc Natl Acad Sci USA 105: 19678-19683, 2008.

23. Lal A, Navarro F, Maher CA, et al: miR-24 Inhibits cell proliferation by targeting E2F2, MYC, and other cell-cycle genes via binding to 'seedless' 3'UTR microRNA recognition elements. Mol Cell 35: 610-625, 2009.

24. Nelson KM and Weiss GJ: MicroRNAs and cancer: past, present, and potential future. Mol Cancer Ther 7: 3655-3660, 2008.

25. Hu X, Guo J, Zheng L, et al: The heterochronic microRNA let-7 inhibits cell motility by regulating the genes in the actin cytoskeleton pathway in breast cancer. Mol Cancer Res 11: 240-250, 2013.

26. Yang Q, Jie Z, Cao H, Greenlee AR, Yang C, Zou F and Jiang Y: Low-level expression of let-7a in gastric cancer and its involvement in tumorigenesis by targeting $\mathrm{RAB} 40 \mathrm{C}$. Carcinogenesis 32: 713-722, 2011.

27. Chen $\mathrm{C}$ and Wells AD: Comparative analysis of E2F family member oncogenic activity. PLoS One 2: e912, 2007.

28. Lin PC, Chiu YL, Banerjee S, et al: Epigenetic repression of miR-31 disrupts androgen receptor homeostasis and contributes to prostate cancer progression. Cancer Res 73: 1232-1244, 2013.

29. Fujiwara K, Yuwanita I, Hollern DP and Andrechek ER: Prediction and genetic demonstration of a role for activator E2Fs in Myc-induced tumors. Cancer Res 71: 1924-1932, 2011.

30. Okamoto OK, Oba-Shinjo SM, Lopes L and Nagahashi Marie SK: Expression of HOXC9 and E2F2 are up-regulated in $\mathrm{CD} 33^{+}$cells isolated from human astrocytomas and associate with transformation of human astrocytes. Biochim Biophys Acta 1769: 437-442, 2007.

31. Dong Q, Meng P, Wang T, et al: MicroRNA let-7a inhibits proliferation of human prostate cancer cells in vitro and in vivo by targeting E2F2 and CCND2. PLoS One 5: e10147, 2010.

32. Sharma N, Timmers C, Trikha P, Saavedra HI, Obery A and Leone G: Control of the p53-p21CIP1 Axis by E2f1, E2f2, and E2f3 is essential for G1/S progression and cellular transformation. J Biol Chem 281: 36124-36131, 2006.

33. Nguyen-Vu T, Vedin LL, Liu K, et al: Liver x receptor ligands disrupt breast cancer cell proliferation through an E2F-mediated mechanism. Breast Cancer Res 15: R51, 2013. 